\title{
DOl: https://doi.org/10.24297/jam.v21i.9166
}

\section{W-Power N-Binormal Operator on Hilbert Space}

Alaa Hussein Mohammed

Department of Mathematics, College of Education, University of Al-Qadisiyah, Diwaniya-Iraq

alaa.hussein@qu.edu

\begin{abstract}
In this paper we present a new class of operators on Hilbert space called w-power n-binormal
\end{abstract} operator. We study this operator and give some properties of it.

Keywords: Normal operator, Binormal operator, Hilbert space.

Introduction: Consider $\mathrm{B}(\mathrm{H})$ be the algebra of all bounded linear operators on Hilbert space $\mathrm{H}$. An operator $\mathrm{S}$ called normal if $S^{*} S=S S^{*}$.In [2] Campbell, Stephen ,L. introduce the class binormal of operator which is defined as $S^{*} S S S^{*}=S S^{*} S^{*} S . . \ln [4]$ Panayappan, S. and Sivamani give a new class of operators called $\mathrm{n}$-binormal and it is defined as $S^{*} S^{n} S^{n} S^{*}=S^{n} S^{*} S^{*} S^{n}$. In this paper we defied a new class of operators on Hilbert space as $\left(S^{w}\right)^{*} S^{n} S^{n}\left(S^{w}\right)^{*}=S^{n}\left(S^{w}\right)^{*}\left(S^{w}\right)^{*} S^{n}$ called w-power n-binormal operator and study some properties of it.

\section{Main Result}

Definition 1.1 Let $S$ be bounded operator. $S$ is called w-power $n$-binormal operator if and only if $\left(S^{w}\right)^{*} S^{n} S^{n}\left(S^{w}\right)^{*}=S^{n}\left(S^{w}\right)^{*}\left(S^{w}\right)^{*} S^{n}$, where $\mathrm{w}, \mathrm{n}$ are nonnegative integer .

Example 1.2 : Let $\mathrm{S}$ be a weighted shift operator of non-zero weights $\left\{\beta_{r}\right\}_{r=0}^{\infty}$.then $\mathrm{S}$ is w-power $\mathrm{n}$-binormal operator if and only if

$\left(\overline{\beta_{r-1}} \ldots \overline{\beta_{r-w}}\right)\left(\beta_{r-w} \ldots \beta_{r-w+n-1}\right)\left(\beta_{r-w+n} \ldots \beta_{r-w+2 n-1}\right)\left(\overline{\beta_{r-w+2 n-1}} \ldots \overline{\beta_{r-2 w+2 n}}\right) z_{r-2 w+2 n}=$ $\left(\beta_{r} \ldots \beta_{r+n-1}\right)\left(\overline{\beta_{r+n-1}} \ldots \overline{\beta_{r+n-w}}\right)\left(\overline{\beta_{r+n-w-1}} \ldots \overline{\beta_{r+n-2 w}}\right)\left(\beta_{r+2 n-2 w} \ldots \beta_{r+2 n-2 w-1}\right) z_{r+2 n-2 w}$

Proof: Suppose $\left\{z_{r}\right\}_{r=0}^{\infty}$ be orthogonal basis of H. Hence $S z_{r}=\beta_{r} z_{r+1}$,

$$
\begin{aligned}
& S^{*} z_{r}=\overline{\beta_{r-1}} z_{r-1} \quad, S^{n} z_{r}=\left(\beta_{r} \ldots \beta_{r+n-1}\right) z_{r+n} \text { and } \\
& \left(S^{w}\right)^{*} z_{r}=\left(S^{*}\right)^{w} z_{r}=\left(\overline{\beta_{r-1}} \ldots \overline{\beta_{r-w}}\right) z_{r-w} \\
& \left(S^{w}\right)^{*} S^{n} S^{n}\left(S^{w}\right)^{*} z_{r}=\left(S^{w}\right)^{*} S^{n}\left(\overline{\beta_{r-1}} \ldots \overline{\beta_{r-w}}\right) S^{n} z_{r-w} \\
& =\left(S^{w}\right)^{*} S^{n}\left(\overline{\beta_{r-1}} \ldots \overline{\beta_{r-w}}\right)\left(\beta_{r-w} \ldots \beta_{r-w+n-1}\right) z_{r-w+n} \\
& =\left(\overline{\beta_{r-1}} \ldots \overline{\beta_{r-w}}\right)\left(\beta_{r-w} \ldots \beta_{r-w+n-1}\right)\left(\beta_{r-w+n} \ldots \beta_{r-w+2 n-1}\right)\left(\overline{\beta_{r-w+2 n-1}} \ldots \overline{\beta_{r-2 w+2 n}}\right) z_{r-2 w+2 n} \\
& S^{n}\left(S^{w}\right)^{*}\left(S^{w}\right)^{*} S^{n} z_{r}=S^{n}\left(S^{w}\right)^{*}\left(\beta_{r} \ldots \beta_{r+n-1}\right)\left(S^{w}\right)^{*} z_{r+n} \\
& =S^{n}\left(S^{w}\right)^{*}\left(\beta_{r} \ldots \beta_{r+n-1}\right)\left(\overline{\beta_{r+n-1}} \ldots \overline{\beta_{r+n-w}}\right) z_{r+n-w} \\
& =S^{n}\left(\beta_{r} \ldots \beta_{r+n-1}\right)\left(\overline{\beta_{r+n-1}} \ldots \overline{\beta_{r+n-w}}\right)\left(\overline{\beta_{r+n-w-1}} \ldots \overline{\beta_{r+n-2 w}}\right) z_{r+n-2 w} \\
& =\left(\beta_{r} \ldots \beta_{r+n-1}\right)\left(\overline{\beta_{r+n-1}} \ldots \overline{\beta_{r+n-w}}\right)\left(\overline{\beta_{r+n-w-1}} \ldots \overline{\beta_{r+n-2 w}}\right)\left(\beta_{r+2 n-2 w} \ldots \beta_{r+2 n-2 w-1}\right) z_{r+2 n-2 w}
\end{aligned}
$$

Hence, $\mathrm{S}$ is $\mathrm{w}$-power $\mathrm{n}$-binormal operator if and only if

$$
\begin{aligned}
& \left(\overline{\beta_{r-1}} \ldots \overline{\beta_{r-w}}\right)\left(\beta_{r-w} \ldots \beta_{r-w+n-1}\right)\left(\beta_{r-w+n} \ldots \beta_{r-w+2 n-1}\right)\left(\overline{\beta_{r-w+2 n-1}} \ldots \overline{\beta_{r-2 w+2 n}}\right) z_{r-2 w+2 n}
\end{aligned}
$$

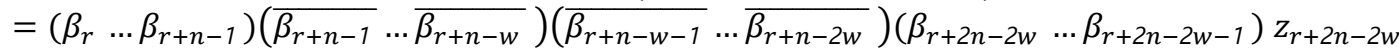

Proposition 1.3 Suppose $\mathrm{S}$ be abounded operator on $\mathrm{H}$, then it is be w-power $\mathrm{n}$-binormal operator if and only if $\mathrm{S}$ is a $\mathrm{n}$-power $\mathrm{w}$-binormal operator.

Proof: Let $S$ be w-power n-binormal operator then $\left(S^{w}\right)^{*} S^{n} S^{n}\left(S^{w}\right)^{*}=S^{n}\left(S^{w}\right)^{*}\left(S^{w}\right)^{*} S^{n}$. Therefore, we need to prove that $\mathrm{S}$ is a $\mathrm{n}$-power $\mathrm{w}$-binormal operator. 
$\left(S^{n}\right)^{*} S^{w} S^{w}\left(S^{n}\right)^{*}=\left[\left[\left(S^{n}\right)^{*} S^{w} S^{w}\left(S^{n}\right)^{*}\right]^{*}\right]^{*}$

$$
\begin{gathered}
=\left[\left[S^{w}\left(S^{n}\right)^{*}\right]^{*}\left[\left(S^{n}\right)^{*} S^{w}\right]^{*}\right]^{*} \\
=\left[\left[\left(S^{n}\right)^{*} S^{w}\right]^{*}\right]^{*}\left[\left[S^{w}\left(S^{n}\right)^{*}\right]^{*}\right]^{*} \\
=\left[\left(S^{w}\right)^{*} S^{n}\right]^{*}\left[S^{n}\left(S^{w}\right)^{*}\right]^{*}
\end{gathered}
$$

$=\left[S^{n}\left(S^{w}\right)^{*}\left(S^{w}\right)^{*} S^{n}\right]^{*}$, since $S$ w-power n-binormal operator

$$
\begin{gathered}
=\left[\left(S^{w}\right)^{*} S^{n} S^{n}\left(S^{w}\right)^{*}\right]^{*} \\
=\left[S^{n}\left(S^{w}\right)^{*}\right]^{*}\left[\left(S^{w}\right)^{*} S^{n}\right]^{*} \\
=S^{w}\left(S^{n}\right)^{*}\left(S^{n}\right)^{*} S^{w}
\end{gathered}
$$

Thus, $\mathrm{S}$ is a $\mathrm{n}$-power $\mathrm{w}$-binormal operator. The convers is similarly.

Definition 1.4 [3]: If $A, B$ are bounded operator on Hilbert space $H$. Then $A, B$ are unitary equivalent if there is an isomorphism $U: H \rightarrow H$ such that $B=U A U^{*}$.

Proposition 1.5 If $\mathrm{S}$ is w-power n-binormal operator,

1.- then $S^{*}$ is w-power $\mathrm{n}$-binormal operator.

2.- If $S^{-1}$ exist then, $S^{-1}$ is w-power n-binormal operator.

3.- If $T \in B(H)$ is unitary equivalent to $\mathrm{S}$ then $\mathrm{T}$ is $\mathrm{w}$-power $\mathrm{n}$-binormal operator.

\section{Proof}

1. Since $S$ is w-power n-binormal operator, then $\left(S^{w}\right)^{*} S^{n} S^{n}\left(S^{w}\right)^{*}=S^{n}\left(S^{w}\right)^{*}\left(S^{w}\right)^{*} S^{n}$.

$\left(\left(S^{*}\right)^{w}\right)^{*}\left(S^{*}\right)^{n}\left(S^{*}\right)^{n}\left(\left(S^{*}\right)^{w}\right)^{*}=S^{w}\left(S^{n}\right)^{*}\left(S^{n}\right)^{*} S^{w}$ By above proposition we have,

$$
\begin{aligned}
& =\left(S^{*}\right)^{w} S^{n} S^{n}\left(S^{*}\right)^{w} \\
& =\left(S^{*}\right)^{n} S^{w} S^{w}\left(S^{*}\right)^{n} \\
& =\left(S^{*}\right)^{n}\left(\left(S^{*}\right)^{*}\right)^{w}\left(\left(S^{*}\right)^{*}\right)^{w}\left(S^{*}\right)^{n} \\
& =\left(S^{*}\right)^{n}\left(\left(S^{*}\right)^{w}\right)^{*}\left(\left(S^{*}\right)^{w}\right)^{*}\left(S^{*}\right)^{n}
\end{aligned}
$$

Hence, $S^{*}$ is w-power n-binormal operator.

2. Consider $\left(\left(S^{-1}\right)^{w}\right)^{*}\left(S^{-1}\right)^{n}\left(S^{-1}\right)^{n}\left(\left(S^{-1}\right)^{w}\right)^{*}$

$$
\begin{gathered}
=\left(\left(S^{*}\right)^{w}\right)^{-1}\left(S^{n}\right)^{-1}\left(S^{n}\right)^{-1}\left(\left(S^{*}\right)^{w}\right)^{-1} \\
=\left[S^{n}\left(S^{*}\right)^{w}\right]^{-1}\left[\left(S^{*}\right)^{w} S^{n}\right]^{-1}
\end{gathered}
$$

$=\left[\left(S^{*}\right)^{w} S^{n} S^{n}\left(S^{*}\right)^{w}\right]^{-1}$, by above proposition

$$
\begin{gathered}
=\left[S^{n}\left(S^{w}\right)^{*}\left(S^{w}\right)^{*} S^{n}\right]^{-1} \\
=\left[S^{n}\left(S^{*}\right)^{w}\right]^{-1}\left[S^{n}\left(S^{*}\right)^{w}\right]^{-1} \\
=\left(S^{n}\right)^{-1}\left(\left(S^{*}\right)^{w}\right)^{-1}\left(\left(S^{*}\right)^{w}\right)^{-1}\left(S^{n}\right)^{-1} \\
=\left(S^{-1}\right)^{n}\left(\left(S^{-1}\right)^{w}\right)^{*}\left(\left(S^{-1}\right)^{w}\right)^{*}\left(S^{-1}\right)^{n}
\end{gathered}
$$

Hence, $S^{-1}$ is w-power n-binormal operator.

3. Since $\mathrm{T}$ is unitary equivalent to $\mathrm{S}$ then $T=U S U^{*}$, therefore $\left(U S U^{*}\right)^{n}=U S^{n} U^{*}$

$$
\begin{gathered}
\left(T^{w}\right)^{*} T^{n} T^{n}\left(T^{w}\right)^{*}=\left(\left(U S U^{*}\right)^{w}\right)^{*}\left(U S U^{*}\right)^{n}\left(U S U^{*}\right)^{n}\left(\left(U S U^{*}\right)^{w}\right)^{*} \\
=\left(U S^{w} U^{*}\right)^{*}\left(U S^{n} U^{*}\right)\left(U S^{n} U^{*}\right)\left(U S^{w} U^{*}\right)^{*} \\
=\left(U\left(S^{w}\right)^{*} U^{*}\right)\left(U S^{n} U^{*}\right)\left(U S^{n} U^{*}\right)\left(U\left(S^{w^{*}}\right) U^{*}\right) \\
=U\left(S^{w}\right)^{*} S^{n} S^{n}\left(S^{w}\right)^{*} U^{*}
\end{gathered}
$$




$$
\begin{gathered}
=U S^{n} U^{*} U\left(S^{w}\right)^{*} U^{*} U\left(S^{w}\right)^{*} U^{*} U S^{n} U^{*} \\
=\left(U S^{n} U^{*}\right)\left(U S^{w} U^{*}\right)^{*}\left(U S^{w} U^{*}\right)^{*}\left(U S^{n} U^{*}\right) \\
=\left(U S U^{*}\right)^{n}\left(\left(U S U^{*}\right)^{w}\right)^{*}\left(\left(U S U^{*}\right)^{w}\right)^{*}\left(U S U^{*}\right)^{n}=T^{n}\left(T^{w}\right)^{*}\left(T^{w}\right)^{*} T^{n}
\end{gathered}
$$

Hence $\mathrm{T}$ is w-power n-binormal operator

Proposition 1.6 Let $\mathrm{S}$ be a bounded operator. If $\mathrm{S}$ is $\mathrm{w}$-power $\mathrm{n}$-binormal operator then $S^{n w}$ is binormal operator.

Proof: Suppose that $\mathrm{S}$ is w-power n-binormal operator then $\left(S^{w}\right)^{*} S^{n} S^{n}\left(S^{w}\right)^{*}=S^{n}\left(S^{w}\right)^{*}\left(S^{w}\right)^{*} S^{n}$ it is clear that $\left(S^{m}\right)^{*}=\left(S^{*}\right)^{m}$ for each nonnegative integer $\mathrm{m}$.

$$
\begin{aligned}
& \left(S^{n w}\right)^{*} S^{n w} S^{n w}\left(S^{n w}\right)^{*}=\left(\left(S^{w}\right)^{n}\right)^{*} S^{n w} S^{n w}\left(\left(S^{w}\right)^{n}\right)^{*} \\
& \underbrace{=\left(S^{w} S^{w} \ldots S^{w}\right)^{*}}_{n \text {-times }} \underbrace{\left(S^{n} S^{n} \ldots S^{n}\right)}_{m \text {-times }} \underbrace{\left(S^{n} S^{n} \ldots S^{n}\right)}_{m \text {-times }} \underbrace{\left(S^{w} S^{w} \ldots S^{w}\right)^{*}}_{n \text {-times }} \\
& \underbrace{=\left(S^{w}\right)^{*}\left(S^{w}\right)^{*} \ldots\left(S^{w}\right)^{*}}_{n \text {-times }} \underbrace{\left(S^{n} S^{n} \ldots S^{n}\right)}_{m \text {-times }} \underbrace{\left(S^{n} S^{n} \ldots S^{n}\right)}_{m-\text { times }} \underbrace{\left(S^{w}\right)^{*}\left(S^{w}\right)^{*} \ldots\left(S^{w}\right)^{*}}_{n \text {-times }} \\
& =\left(S^{w}\right)^{*}\left(S^{w}\right)^{*} \ldots S^{n}\left(S^{w}\right)^{*} S^{n} S^{n} \ldots S^{n} \cdot S^{n} S^{n} \ldots\left(S^{w}\right)^{*} S^{n} \cdot\left(S^{w}\right)^{*}\left(S^{w}\right)^{*} \ldots\left(S^{w}\right)^{*} \\
& \vdots \\
& =\underbrace{\left(S^{n} S^{n} \ldots S^{n}\right)}_{m-\text { times }} \underbrace{\left(S^{w}\right)^{*}\left(S^{w}\right)^{*} \ldots\left(S^{w}\right)^{*}}_{n-\text { times }} \underbrace{\left(S^{n} S^{n} \ldots S^{n}\right)}_{n-\text { times }} \underbrace{\left(S^{w} S^{w} \ldots S^{w}\right)^{*}}_{n-\text { times }} \underbrace{\left(S^{w} S^{w} \ldots S^{w}\right)^{*}}_{n-\text { times }} \underbrace{\left(S^{n} S^{n} \ldots S^{n}\right)}_{m-\text { times }} \\
& =S^{n w}\left(\left(S^{w}\right)^{n}\right)^{*}\left(\left(S^{w}\right)^{n}\right)^{*} S^{n w} \\
& =S^{n w}\left(S^{n w}\right)^{*}\left(S^{n w}\right)^{*} S^{n w} .
\end{aligned}
$$

Hence, $S^{n w}$ is binormal operator.

Theorem 1.7 The set of all w-power n-binormal operators on $H$ is a closed subset of $B(H)$ under scalar multiplication.

Proof: Let

$$
\mathrm{W}(\mathrm{H})=\{S \in B(H): S \text { is } \mathrm{w} \text { - power } \mathrm{n} \text { - binormal operator on } \mathrm{H} \text { for some nonnegative integer } \mathrm{w}\}
$$

Let $S \in W(H)$ then we have $S$ is w-power n-binormal operator and thus $\left(S^{w}\right)^{*} S^{n} S^{n}\left(S^{w}\right)^{*}=S^{n}\left(S^{w}\right)^{*}\left(S^{w}\right)^{*} S^{n}$.

Let $\gamma$ be a scalar, hence

$$
\begin{aligned}
\left((\gamma S)^{w}\right)^{*}(\gamma S)^{n}(\gamma S)^{n}\left((\gamma S)^{w}\right)^{*}=(\bar{\gamma})^{w}\left(S^{w}\right)^{*} \gamma^{n} S^{n} \gamma^{n} S^{n}(\bar{\gamma})^{w}\left(S^{w}\right)^{*} & \\
& =(\bar{\gamma})^{w} \gamma^{n} \gamma^{n}(\bar{\gamma})^{w}\left(S^{w}\right)^{*} S^{n} S^{n}\left(S^{w}\right)^{*} \\
& =(\bar{\gamma})^{w} \gamma^{n} \gamma^{n}(\bar{\gamma})^{w} S^{n}\left(S^{w}\right)^{*}\left(S^{w}\right)^{*} S^{n}
\end{aligned}
$$

$=\gamma^{n} S^{n}(\bar{\gamma})^{w}\left(S^{w}\right)^{*}(\bar{\gamma})^{w}\left(S^{w}\right)^{*} \gamma^{n} S^{n}$

$$
=(\gamma S)^{n}\left((\gamma S)^{w}\right)^{*}\left((\gamma S)^{w}\right)^{*}(\gamma S)^{n}
$$

Thus $\gamma S \in W(H)$,

Let $S_{k}$ be a sequence in $W(H)$ and converge to $S$, then we can get that

$\left\|\left(\square^{\square}\right)^{*} \square^{\square} \square^{\square}\left(\square^{\square}\right)^{*}-\square^{\square}\left(\square^{\square}\right)^{*}\left(\square^{\square}\right)^{*} \square^{\square}\right\|$

$$
=\left\|\left(S^{w}\right)^{*} S^{n} S^{n}\left(S^{w}\right)^{*}-\left(S_{k}^{w}\right)^{*} S_{k}^{n} S_{k}^{n}\left(S_{k}^{w}\right)^{*}+S_{k}^{n}\left(S_{k}^{w}\right)^{*}\left(S_{k}^{w}\right)^{*} S_{k}^{n}-S^{n}\left(S^{w}\right)^{*}\left(S^{w}\right)^{*} S^{n}\right\|
$$

$\leq\left\|\left(S^{w}\right)^{*} S^{n} S^{n}\left(S^{w}\right)^{*}-\left(S_{k}^{w}\right)^{*} S_{k}^{n} S_{k}^{n}\left(S_{k}^{w}\right)^{*}\right\|+\left\|S_{k}^{n}\left(S_{k}^{w}\right)^{*}\left(S_{k}^{w}\right)^{*} S_{k}^{n}-S^{n}\left(S^{w}\right)^{*}\left(S^{w}\right)^{*} S^{n}\right\| \rightarrow 0$ as $k \rightarrow \infty$.

Hence, $\left(S^{w}\right)^{*} S^{n} S^{n}\left(S^{w}\right)^{*}=S^{n}\left(S^{w}\right)^{*}\left(S^{w}\right)^{*} S^{n}$ therefore $S \in W(H)$. 
Then, $W(H)$ is closed subset.

Theorem 1.8: If $\mathrm{R}$ and $\mathrm{S}$ are w-power $\mathrm{n}$-binormal operators on $\mathrm{H}$, and let $\mathrm{S}$ commute with $\mathrm{R}$ then $(S R)$ is wpower $\mathrm{n}$-binormal operator on $\mathrm{H}$.

Proof:

$$
\begin{aligned}
\left(\left(S R^{w}\right)\right)^{*}(S R)^{n}(S R)^{n}\left((S R)^{w}\right)^{*}=\left(R^{w}\right)^{*}\left(S^{w}\right)^{*}(R)^{n}(S)^{n}(R)^{n}(S)^{n}\left(R^{w}\right)^{*}\left(S^{w}\right)^{*} \\
=\left(R^{w}\right)^{*}\left(S^{w}\right)^{*}(S)^{n}(S)^{n}(R)^{n}(R)^{n}\left(S^{w}\right)^{*}\left(R^{w}\right)^{*} \\
=\left(R^{w}\right)^{*}\left(S^{w}\right)^{*}(S)^{n}(S)^{n}(R)^{n}\left(S^{w}\right)^{*}(R)^{n}\left(R^{w}\right)^{*} \\
=\left(R^{w}\right)^{*}(S)^{n}\left(S^{w}\right)^{*}(S)^{n}\left(S^{w}\right)^{*}(R)^{n}(R)^{n}\left(R^{w}\right)^{*} \\
=(S)^{n}\left(R^{w}\right)^{*}\left(S^{w}\right)^{*}(S)^{n}\left(S^{w}\right)^{*}(R)^{n}(R)^{n}\left(R^{w}\right)^{*} \\
=(S)^{n}\left(R^{w}\right)^{*}\left(S^{w}\right)^{*}(S)^{n}(R)^{n}\left(S^{w}\right)^{*}(R)^{n}\left(R^{w}\right)^{*} \\
=(S)^{n}\left(R^{w}\right)^{*}\left(S^{w}\right)^{*}(R)^{n}(S)^{n}\left(S^{w}\right)^{*}(R)^{n}\left(R^{w}\right)^{*} \\
=(S)^{n}\left(R^{w}\right)^{*}(R)^{n}\left(S^{w}\right)^{*}(S)^{n}\left(S^{w}\right)^{*}(R)^{n}\left(R^{w}\right)^{*} \\
=(S)^{n}(R)^{n}\left(R^{w}\right)^{*}\left(S^{w}\right)^{*}(S)^{n}\left(S^{w}\right)^{*}(R)^{n}\left(R^{w}\right)^{*} \\
=(S)^{n}(R)^{n}\left(R^{w}\right)^{*}\left(S^{w}\right)^{*}\left(S^{w}\right)^{*}(S)^{n}(R)^{n}\left(R^{w}\right)^{*} \\
=(S)^{n}(R)^{n}\left(R^{w}\right)^{*}\left(S^{w}\right)^{*}\left(S^{w}\right)^{*}\left(R^{w}\right)^{*}(S)^{n}(R)^{n} \\
=(S)^{n}(R)^{n}\left(R^{w}\right)^{*}\left(S^{w}\right)^{*}\left(R^{w}\right)^{*}\left(S^{w}\right)^{*}(R)^{n}(S)^{n} \\
=(S R)^{n}\left(\left(S R^{w}\right)\right)^{*}\left((S R)^{w}\right)^{*}(S R)^{n}
\end{aligned}
$$

Theorem 1.9: Let $S_{1}, S_{2}, \ldots, S_{k}$, are w-power $\mathrm{n}$-binormal operators on $\mathrm{H}$. Then the direct sum $\left(S_{1} \oplus S_{2} \oplus \ldots \oplus S_{k}\right)$ is w-power $\mathrm{n}$-binormal operator on $\mathrm{H}$.

Proof: Since every operator of $S_{1}, S_{2}, \ldots, S_{k}$ is w-power n-binormal, then

$$
\begin{gathered}
\left(S_{l}{ }^{w}\right)^{*} S_{l}{ }^{n} S_{l}{ }^{n}\left(S_{l}{ }^{w}\right)^{*}=S_{l}{ }^{n}\left(S_{l}{ }^{w}\right)^{*}\left(S_{l}{ }^{w}\right)^{*} S_{l}{ }^{n} \text { for all } l=1,1, \ldots, k \\
\begin{array}{c}
\left(\left(S_{1} \oplus S_{2} \oplus \ldots \oplus S_{k}\right)^{w}\right)^{*}\left(S_{1} \oplus S_{2} \oplus \ldots \oplus S_{k}\right)^{n}\left(S_{1} \oplus S_{2} \oplus \ldots \oplus S_{k}\right)^{n}\left(\left(S_{1} \oplus S_{2} \oplus \ldots \oplus S_{k}\right)^{w}\right)^{*} \\
=\left(S_{1}{ }^{w} \oplus S_{2}{ }^{w} \oplus \ldots \oplus S_{k}{ }^{w}\right)^{*}\left(S_{1}{ }^{n} \oplus S_{2}{ }^{n} \oplus \ldots \oplus S_{k}{ }^{n}\right)\left(S_{1}{ }^{n} \oplus S_{2}{ }^{n} \oplus \ldots \oplus S_{k}{ }^{n}\right)\left(S_{1}{ }^{w} \oplus S_{2}{ }^{w} \oplus \ldots \oplus S_{k}{ }^{w}\right)^{*} \\
=\left[\left(S_{1}{ }^{w}\right)^{*} \oplus\left(S_{2}{ }^{w}\right)^{*} \oplus \ldots \oplus\left(S_{k}{ }^{w}\right)^{*}\right]\left(S_{1}{ }^{n} \oplus S_{2}{ }^{n} \oplus \ldots \oplus S_{k}{ }^{n}\right)\left(S_{1}{ }^{n} \oplus S_{2}{ }^{n} \oplus \ldots \oplus S_{k}{ }^{n}\right)\left[\left(S_{1}{ }^{w}\right)^{*} \oplus\left(S_{2}{ }^{w}\right)^{*} \oplus \ldots \oplus\left(S_{k}{ }^{w}\right)^{*}\right] \\
=\left(S_{1}{ }^{w}\right)^{*} S_{1}{ }^{n} S_{1}{ }^{n}\left(S_{1}{ }^{w}\right)^{*} \oplus\left(S_{2}{ }^{w}\right)^{*} S_{2}{ }^{n} S_{2}{ }^{n}\left(S_{2}{ }^{w}\right)^{*} \oplus \ldots \oplus\left(S_{k}{ }^{w}\right)^{*} S_{k}{ }^{n} S_{k}{ }^{n}\left(S_{k}{ }^{w}\right)^{*} \\
=S_{1}{ }^{n}\left(S_{1}{ }^{w}\right)^{*}\left(S_{1}{ }^{w}\right)^{*} S_{1}{ }^{n} \oplus S_{2}{ }^{n}\left(S_{2}{ }^{w}\right)^{*}\left(S_{2}{ }^{w}\right)^{*} S_{2}{ }^{n} \oplus \ldots \oplus S_{k}{ }^{n}\left(S_{k}{ }^{w}\right)^{*}\left(S_{k}{ }^{w}\right)^{*} S_{k}{ }^{n} \\
=\left(S_{1}{ }^{n} \oplus S_{2}{ }^{n} \oplus \ldots \oplus S_{k}{ }^{n}\right)\left[\left(S_{1}{ }^{w}\right)^{*} \oplus\left(S_{2}{ }^{w}\right)^{*} \oplus \ldots \oplus\left(S_{k}{ }^{w}\right)^{*}\right]\left[\left(S_{1}{ }^{w}\right)^{*} \oplus\left(S_{2}{ }^{w}\right)^{*} \oplus \ldots \oplus\left(S_{k}{ }^{w}\right)^{*}\right]\left(S_{1}{ }^{n} \oplus S_{2}{ }^{n} \oplus \ldots \oplus S_{k}{ }^{n}\right) \\
=\left(S_{1}{ }^{n} \oplus S_{2}{ }^{n} \oplus \ldots \oplus S_{k}{ }^{n}\right)\left(S_{1}{ }^{w} \oplus S_{2}{ }^{w} \oplus \ldots \oplus S_{k}{ }^{w}\right)^{*}\left(S_{1}{ }^{w} \oplus S_{2}{ }^{w} \oplus \ldots \oplus S_{k}{ }^{w}\right)^{*}\left(S_{1}{ }^{n} \oplus S_{2}{ }^{n} \oplus \ldots \oplus S_{k}{ }^{n}\right) \\
=\left(S_{1} \oplus S_{2} \oplus \ldots \oplus S_{k}\right)^{n}\left(\left(S_{1} \oplus S_{2} \oplus \ldots \oplus S_{k}\right)^{w}\right)^{*}\left(\left(S_{1} \oplus S_{2} \oplus \ldots \oplus S_{k}\right)^{w}\right)^{*}\left(S_{1} \oplus S_{2} \oplus \ldots \oplus S_{k}\right)^{n}
\end{array}
\end{gathered}
$$

Thus, $\left(S_{1} \oplus S_{2} \oplus \ldots \oplus S_{k}\right)$ is w-power n-binormal operator on $\mathrm{H}$.

Theorem 1.10: Let $S_{1}, S_{2}, \ldots, S_{k}$, are w-power $\mathrm{n}$-binormal operators on $\mathrm{H}$. Then the tenser product $\left(S_{1} \otimes S_{2} \otimes \ldots \otimes S_{k}\right)$ is w-power n-binormal operator on $\mathrm{H}$.

Proof: Since every operator of $S_{1}, S_{2}, \ldots, S_{k}$ is w-power n-binormal, then

$\left(S_{l}{ }^{w}\right)^{*} S_{l}{ }^{n} S_{l}{ }^{n}\left(S_{l}{ }^{w}\right)^{*}=S_{l}{ }^{n}\left(S_{l}{ }^{w}\right)^{*}\left(S_{l}{ }^{w}\right)^{*} S_{l}{ }^{n}$ for all $l=1,1, \ldots, k$

$\left(\left(S_{1} \otimes S_{2} \otimes \ldots \otimes S_{k}\right)^{w}\right)^{*}\left(S_{1} \otimes S_{2} \otimes \ldots \otimes S_{k}\right)^{n}\left(S_{1} \otimes S_{2} \otimes \ldots \otimes S_{k}\right)^{n}\left(\left(S_{1} \otimes S_{2} \otimes \ldots \otimes S_{k}\right)^{w}\right)^{*}\left(\boldsymbol{x}_{\mathbf{1}} \otimes \boldsymbol{x}_{2} \otimes \ldots \otimes \boldsymbol{x}_{\mathbf{k}}\right)$

$=\left(S_{1}{ }^{w} \otimes S_{2}{ }^{w} \otimes \ldots \otimes S_{k}{ }^{w}\right)^{*}\left(S_{1}{ }^{n} \otimes S_{2}{ }^{n} \otimes \ldots \otimes S_{k}{ }^{n}\right)\left(S_{1}{ }^{n} \otimes S_{2}{ }^{n} \otimes \ldots \otimes S_{k}{ }^{n}\right)\left(S_{1}{ }^{w} \otimes S_{2}{ }^{w} \otimes \ldots \otimes S_{k}{ }^{w}\right)^{*}\left(\boldsymbol{x}_{\mathbf{1}} \otimes \boldsymbol{x}_{\mathbf{2}} \otimes \ldots \otimes \boldsymbol{x}_{\mathbf{k}}\right)$ 


$$
\begin{gathered}
=\left[\left(S_{1}{ }^{w}\right)^{*} \otimes\left(S_{2}{ }^{w}\right)^{*} \otimes \ldots \otimes\left(S_{k}{ }^{w}\right)^{*}\right]\left(S_{1}{ }^{n} \otimes S_{2}{ }^{n} \otimes \ldots \otimes S_{k}{ }^{n}\right)\left(S_{1}{ }^{n} \otimes S_{2}{ }^{n} \otimes \ldots \otimes S_{k}{ }^{n}\right)\left[\left(S_{1}{ }^{w}\right)^{*} \otimes\left(S_{2}{ }^{w}\right)^{*} \otimes \ldots \otimes\left(S_{k}{ }^{w}\right)^{*}\right]\left(\boldsymbol{x}_{\mathbf{1}} \otimes \boldsymbol{x}_{\mathbf{2}} \otimes \ldots \otimes \boldsymbol{x}_{\mathbf{k}}\right) \\
=\left(S_{1}{ }^{w}\right)^{*} S_{1}{ }^{n} S_{1}{ }^{n}\left(S_{1}{ }^{w}\right)^{*} \boldsymbol{x}_{\mathbf{1}} \otimes\left(S_{2}{ }^{w}\right)^{*} S_{2}{ }^{n} S_{2}{ }^{n}\left(S_{2}{ }^{w}\right)^{*} \boldsymbol{x}_{\mathbf{2}} \otimes \ldots \otimes\left(S_{k}{ }^{w}\right)^{*} S_{k}{ }^{n} S_{k}{ }^{n}\left(S_{k}{ }^{w}\right)^{*} \boldsymbol{x}_{\mathbf{k}} \\
\quad=S_{1}{ }^{n}\left(S_{1}{ }^{w}\right)^{*}\left(S_{1}{ }^{w}\right)^{*} S_{1}{ }^{n} \boldsymbol{x}_{\mathbf{1}} \otimes S_{2}{ }^{n}\left(S_{2}{ }^{w}\right)^{*}\left(S_{2}{ }^{w}\right)^{*} S_{2}{ }^{n} \boldsymbol{x}_{\mathbf{2}} \otimes \ldots \otimes S_{k}{ }^{n}\left(S_{k}{ }^{w}\right)^{*}\left(S_{k}{ }^{w}\right)^{*} S_{k}{ }^{n} \boldsymbol{x}_{\mathbf{k}} \\
=\left(S_{1}{ }^{n} \otimes S_{2}{ }^{n} \otimes \ldots \otimes S_{k}{ }^{n}\right)\left[\left(S_{1}{ }^{w}\right)^{*} \otimes\left(S_{2}{ }^{w}\right)^{*} \otimes \ldots \otimes\left(S_{k}{ }^{w}\right)^{*}\right]\left[\left(S_{1}{ }^{w}\right)^{*} \otimes\left(S_{2}{ }^{w}\right)^{*} \otimes \ldots \otimes\left(S_{k}{ }^{w}\right)^{*}\right]\left(S_{1}{ }^{n} \otimes S_{2}{ }^{n} \otimes \ldots \otimes S_{k}{ }^{n}\right)\left(\boldsymbol{x}_{\mathbf{1}} \otimes \boldsymbol{x}_{\mathbf{2}} \otimes \ldots \otimes \boldsymbol{x}_{\mathbf{k}}\right) \\
=\left(S_{1}{ }^{n} \otimes S_{2}{ }^{n} \otimes \ldots \otimes S_{k}{ }^{n}\right)\left(S_{1}{ }^{w} \otimes S_{2}{ }^{w} \otimes \ldots \otimes S_{k}{ }^{w}\right)^{*}\left(S_{1}{ }^{w} \otimes S_{2}{ }^{w} \otimes \ldots \otimes S_{k}{ }^{w}\right)^{*}\left(S_{1}{ }^{n} \otimes S_{2}{ }^{n} \otimes \ldots \otimes S_{k}{ }^{n}\right)\left(\boldsymbol{x}_{\mathbf{1}} \otimes \boldsymbol{x}_{\mathbf{2}} \otimes \ldots \otimes \boldsymbol{x}_{\mathbf{k}}\right) \\
\quad=\left(S_{1} \otimes S_{2} \otimes \ldots \otimes S_{k}\right)^{n}\left(\left(S_{1} \otimes S_{2} \otimes \ldots \otimes S_{k}\right)^{w}\right)^{*}\left(\left(S_{1} \otimes S_{2} \otimes \ldots \otimes S_{k}\right)^{w}\right)^{*}\left(S_{1} \otimes S_{2} \otimes \ldots \otimes S_{k}\right)^{n}\left(\boldsymbol{x}_{\mathbf{1}} \otimes \boldsymbol{x}_{\mathbf{2}} \otimes \ldots \otimes \boldsymbol{x}_{\mathbf{k}}\right)
\end{gathered}
$$

Thus, $\left(S_{1} \otimes S_{2} \otimes \ldots \otimes S_{k}\right)$ is w-power n-binormal operator on $\mathrm{H}$.

\section{References:}

1. Berberian, S.K. Introduction to Hilbert space. 1976.Sec. Ed,Chelesa Publishing Com.New York.

2. Campbell,Stephen ,L. 1972.Linear operators for which $T^{*} T$ and $T T^{*}$ commute. 1972. Am-math.Soc.34,177-80.

3. Conway.J.B. A course in functional analysis.1985. New York. Speinger-Verlag

4. Panayappan, S. and Sivamani,N.On n-binormal operators.2012.General Mathematics Notes 10,pp1-8. 